\title{
Preparation and magnetic properties of concentrated magnetic fluids on alcohol and water carrier liquids
}

\author{
Doina Bica ${ }^{\mathrm{a}}$, L. Vékás ${ }^{\mathrm{a}, *}$, M. Rașa ${ }^{\mathrm{b}, 1}$ \\ ${ }^{a}$ Laboratory of Magnetic Fluids, Center for Fundamental and Advanced Technical Research, Romanian Academy-Timisoara Branch, \\ Blvd. Mihai Viteazul 24, 1900 Timisoara, Romania \\ ${ }^{\mathrm{b}}$ Institute for Complex Fluids, "Politehnica" University of Timișoara, Bd. Mihai Viteazul no. 1, 1900 Timișoara, Romania
}

\begin{abstract}
The preparation procedure for very concentrated magnetic fluids on various polar liquids, especially on $\mathrm{C}_{3}-\mathrm{C}_{10}$ (propanol, ..., decanol) alcohol carriers is presented. Double layer sterical stabilization mechanism ensured the high colloidal stability of samples, up to the limiting value of the hydrodynamic volume fraction $(\sim 0.65)$, corresponding to saturation magnetization values of about $70 \mathrm{kA} / \mathrm{m}$. The low-field part of the reduced magnetization curve of the most polar high concentration propanol sample is compared to those corresponding to low and medium concentration waterbased samples.
\end{abstract}

(C) 2002 Elsevier Science B.V. All rights reserved.

Keywords: Polar magnetic fluids; Preparation procedure; Magnetic properties

\section{Introduction}

Stable dispersion of magnetic nanoparticles in polar carriers implies a very careful stabilization procedure, especially if the aim is to attain high magnetization magnetic fluids, when the hydrodynamic volume fraction $\varphi_{\mathrm{h}}$ of particles approaches the upper limit of about 0.65 or when highly polar carriers are envisaged. Various stabilization mechanisms are possible, as described in Refs. [1-4].

The polar carrier liquids, considered in Refs. [1,2,4] and in this work, are the following: high vacuum oils with polar components, weakly polar synthetic oils (diesters), paraffin oil, alcohols, ketones and amines, as well as water. Most of them are of interest for various engineering and biological applications.

Among the polar carriers mentioned above, the $\mathrm{C}_{1}{ }^{-}$ $\mathrm{C}_{10}$ alcohols and water offer a particularly interesting set

\footnotetext{
*Corresponding author.

E-mail address: vekas@flumag2.mec.utt.ro (L. Vékás).

${ }^{1}$ Present address: Van't Hoff Laboratory, Debye Institute, University of Utrecht.
}

of carriers, their degree of polarity varying between large limits.

In Ref. [1] we reported the preparation of medium concentrated $\mathrm{C}_{4}-\mathrm{C}_{9}$-alcohol-based magnetic fluids, while in Ref. [3] Fujita et al. presented a method applied to obtain $\mathrm{C}_{1}-\mathrm{C}_{4}$ alcohols, as well as acetone-based magnetic fluids of low or even very low concentration.

\section{Materials and procedures}

(a) The main steps of the preparation procedure applied for $\mathrm{C}_{3}-\mathrm{C}_{10}$ alcohol carriers are as follows: coprecipitation of $\mathrm{Fe}^{2+}, \mathrm{Fe}^{3+}$ ions (excess of concentrated $\left.\mathrm{NH}_{4} \mathrm{OH} ; \sim 80^{\circ} \mathrm{C}\right) \rightarrow$ subdomain $\mathrm{Fe}_{3} \mathrm{O}_{4}$ nanoparticles - primary sterical stabilization (chemisorbtion of chemically pure oleic acid; $\geqslant 80^{\circ} \mathrm{C}$ ) - phase separationmagnetic decantation $\rightarrow$ monolayer-covered $\mathrm{Fe}_{3} \mathrm{O}_{4}$ nanoparticles + free oleic acid-flocculation/extraction of surfacted particles - dispersion in a light hydrocarbon (repeated/prolonged process) - extraction of excess surfactant $\rightarrow$ nonpolar purified magnetic fluid-flocculation of surfacted particles - secondary stabilization (physical adsorbtion of dodecylbenzensulphonic acid (DBS) and 
redispersion of $\mathrm{Fe}_{3} \mathrm{O}_{4}$ particles in the alcohol carrier liquids listed above $\rightarrow \mathrm{C}_{3}-\mathrm{C}_{10}$-alcohol-based magnetic fluids.

The double layer sterical stabilization method was applied, using chemically pure oleic acid as the primary (chemisorbed) surfactant and technical grade DBS as the secondary (physically adsorbed) surfactant, conducted to very concentrated $\mathrm{C}_{3}-\mathrm{C}_{10}$-alcohol-based samples.

(b) The water-based samples were prepared applying a different procedure: coprecipitation of $\mathrm{Fe}^{2+}, \mathrm{Fe}^{3+}$ ions (excess of concentrated $\mathrm{NH}_{4} \mathrm{OH} ; \sim 80^{\circ} \mathrm{C}$ ) $\rightarrow$ subdomain $\mathrm{Fe}_{3} \mathrm{O}_{4}$ nanoparticles - repeated washing/purificationdouble layer sterical stabilization $\left(\mathrm{DBS}+\mathrm{DBS} ; 80^{\circ} \mathrm{C}\right) \rightarrow$ double layer sterical+electrostatical stabilized $\mathrm{Fe}_{3} \mathrm{O}_{4}$ nanoparticles - dispersion of magnetic nanoparticles in water (ultrasonication)-purification $\rightarrow$ water-based magnetic fluid samples of low and medium concentration.

\section{Results and discussion}

Procedure (a) proved be efficient to obtain very concentrated magnetic fluids, with $\varphi_{\mathrm{h}}$ up to $0.6-0.65$, on various nonaqueous carriers, among them $\mathrm{C}_{3}-\mathrm{C}_{10}$ alcohols, while procedure (b) gave good results in preparing medium concentration water-based samples.

In the case of stabilization procedure (a) the surfactant layers are of different composition. The secondary, physically adsorbed DBS layer has the $\mathrm{SO}_{3} \mathrm{H}$ group towards the dispersion medium. Solvation takes place due to the hydrogen bonds between the $-\mathrm{OH}$ group of alcohol molecules and the $-\mathrm{OH}$ group of $\mathrm{SO}_{3} \mathrm{H}$.

In the case of procedure (b) applied for water-based magnetic fluids, the surfactant layers are identical (DBS + DBS) and the stabilization is mixed, double layer sterical+electrostatical. Indeed, the secondary DBS layer has the groups $-\mathrm{SO}_{3}^{-1}$ oriented towards the dispersion medium and it is covered with hydrated $\mathrm{NH}_{4}^{+}$ ions. Consequently, the degree of stability of waterbased magnetic fluids depends on the $\mathrm{pH}$ value of the medium and the stabilization mechanism is specific only to water as carrier liquid.

The saturation magnetization of the $\mathrm{C}_{3}-\mathrm{C}_{10}$-alcoholbased samples attains $800-900 \mathrm{G}$, the highest values reported up to now for alcohol carriers. The water-based fluid samples obtained have their magnetization up to approx. $400 \mathrm{G}$, but their stability decreases with increasing volume fraction of particles.

The magnetic, magnetooptical, rheological and magnetorheological characterization methods currently applied are presented in Refs. [5-8]. In this work the magnetization curves of the samples are presented to evidence their outstanding properties. The whole relative magnetization $M / M_{\mathrm{s}}=f(H)$ curves of the highest magnetization $\mathrm{C}_{3}-\mathrm{C}_{10}$ samples are given in Fig. 1 $\left(M_{\mathrm{s}} \sim \varphi_{\mathrm{p}}\right.$, where $M_{\mathrm{s}}$ is the saturation magnetization of magnetic fluid and $\varphi_{\mathrm{p}}$ the physical volume fraction of magnetic particles). The low-field part of these curves differentiates between the propanol magnetic fluid, the most polar sample and other alcohol-based magnetic fluids, as shown in Fig. 2. The slight difference evidenced in Fig. 2 between the propanol $\left(\mathrm{C}_{3}\right)$ and the $\left(\mathrm{C}_{4}-\mathrm{C}_{10}\right)$ series magnetic fluids denotes a weak tendency of agglomerate formation in the $\mathrm{C}_{3}$ sample. In Fig. 3 the high concentration propanol-based magnetic fluid is compared to a very diluted and to a medium concentrated water-based sample, especially the last one with a lower degree of stability, specific to water magnetic fluids. The presence of agglomerates in the water-based samples is evidenced by their much greater initial susceptivity, compared to that of the propanol sample, as it follows from the low-field part of magnetization curves (Fig. 3).

As it was shown by sedimentation velocity and electrophoresis investigations for the pentanol magnetic

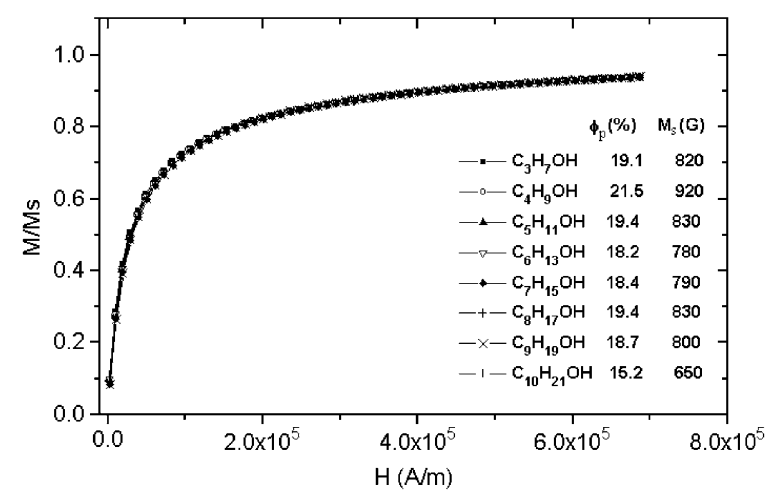

Fig. 1. Reduced magnetization curves of high concentration $\mathrm{C}_{3}-\mathrm{C}_{10}$ alcohol-based magnetic fluids.

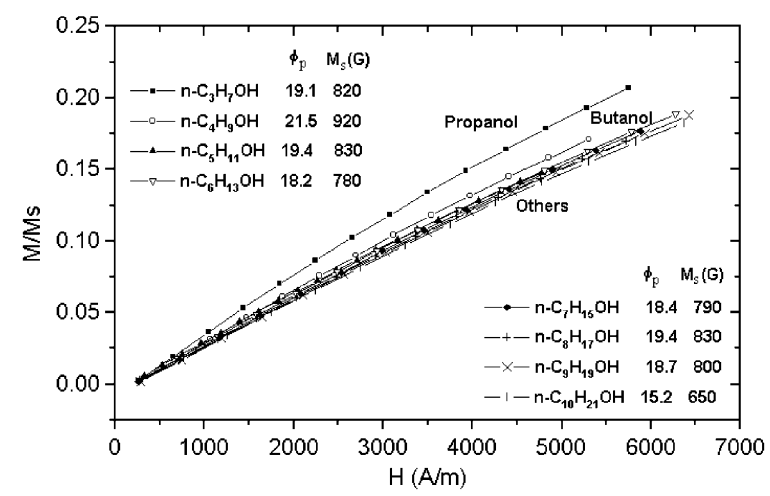

Fig. 2. Low-field reduced magnetization curves of high concentration $\mathrm{C}_{3}-\mathrm{C}_{10}$ alcohol-based magnetic fluids. 


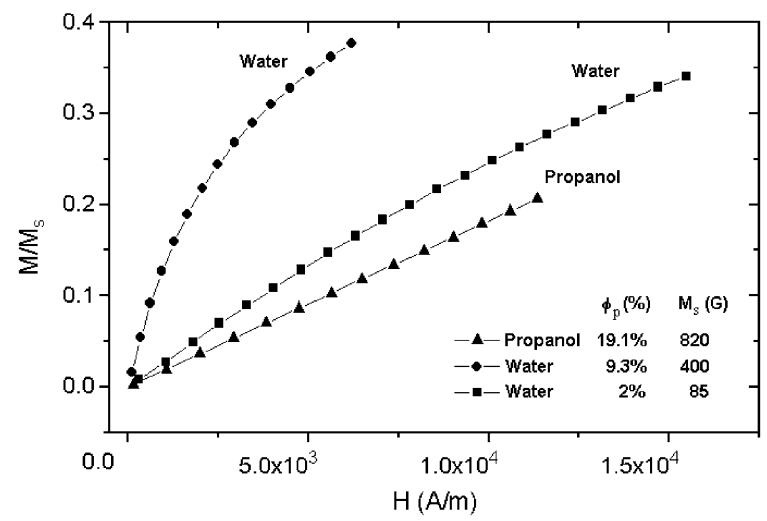

Fig. 3. Low-field reduced magnetization curves of propanol and water-based magnetic fluids.

fluid [9], the high degree of stability could be due, besides sterical, also to the electrostatical repulsion between dispersed nanoparticles, but the origin of electric charges is not the same as in the case of procedure (b) and needs further investigations.

The stabilization of $\mathrm{C}_{3}-\mathrm{C}_{10}$ magnetic fluids was achieved by optimizing the ratio of the mass of secondary surfactant relative to the mass of primary surfactant covered $\mathrm{Fe}_{3} \mathrm{O}_{4}$ nanoparticles. This mass ratio was found to be approximately the same for $\mathrm{C}_{4}-\mathrm{C}_{10}$ alcohols and about $25 \%$ greater for the more polar propanol magnetic fluid.
The series of $\left(\mathrm{C}_{3}-\mathrm{C}_{10}\right)$ alcohol-based magnetic fluid samples of the highest magnetization prepared up to now show excellent colloidal stability.

\section{Acknowledgements}

The support received through the research grants No. 121/1999 and No. 64/2000 of the Romanian Academy, coordinated by Professor Ioan Anton, member of the Romanian Academy, is gratefully acknowledged by the authors.

\section{References}

[1] Doina Bica, L.Vékás, Magnitnaia Gidrodinamika (Magnetohydrodynamics) 30 (1994) 194.

[2] Doina Bica, Romanian Rep. Phys. 47 (1995) 265.

[3] T. Fujita, T. Miyazaki, H. Nishiyama, B. Jeyadevan, J. Magn. Magn. Mater. 201 (1999) 14.

[4] Doina Bica, Romanian Patent, RO 90078, 1985.

[5] M. Rașa, Eur. Phys. J. E 2 (2000) 265.

[6] L. Vékás, M. Rașa, Doina Bica, J. Colloid Interface Sci. 231 (2000) 247.

[7] L. Vékás, Doina Bica, I. Potencz, Daniela Gheorghe, Oana Bălău, M. Rașa, Progr. Colloid Polym. Sci. 117 (2001) 104.

[8] V. Socoliuc, Doina Bica, Progr. Colloid Polym. Sci. 117 (2001) 131.

[9] L.N. Donselaar, A.P. Philipse, J. Suurmond, Langmuir 13 (1997) 6018 